\title{
Porcine Placenta Extract Reduced Wrinkle Formation by Potentiating Epidermal Hydration
}

\section{Akihiro Aioi ${ }^{1,2}{ }^{*}$, Ryuta Muromoto², Sho Mogami1, Megumi Nishikawa1, Shigeyuki Ogawa1, Tadashi Matsuda ${ }^{2}$}

${ }^{1}$ New Business Development Division, Septem-Soken, Osaka, Japan

${ }^{2}$ Department of Immunology, Faculty of Pharmaceutical Sciences, Hokkaido University, Sapporo, Japan

Email: ^a-aioi@septem-so.com

How to cite this paper: Aioi, A., Muromoto, R., Mogami, S., Nishikawa, M., Ogawa, S. and Matsuda, T. (2021) Porcine Placenta Extract Reduced Wrinkle Formation by Potentiating Epidermal Hydration. Journal of Cosmetics, Dermatological Sciences and Applications, 11, 101-109. https://doi.org/10.4236/jcdsa.2021.112011

Received: April 8, 2021

Accepted: June 1, 2021

Published: June 4, 2021

Copyright $\odot 2021$ by author(s) and Scientific Research Publishing Inc. This work is licensed under the Creative Commons Attribution International License (CC BY 4.0).

http://creativecommons.org/licenses/by/4.0/

(c) (i) Open Access

\begin{abstract}
Background: Placenta extract is used as an alternative medicine especially in Asian countries as it is a rich reservoir of diverse bioactive molecules. The effects of placenta extract on skin conditions have been previously reported, however, the mechanism underlying for reduced wrinkle formation remains unclear. Objective: The primary objective of this study was to determine whether the continuous application of porcine placenta extract (PPE) alleviates wrinkle formation in humans and explore the underlying mechanism. Methods: Wrinkle formation, skin hydration, and skin elasticity were measured in 15 volunteers at weeks 0 and 6 after continuous application of a gel containing PPE. The production of type I collagen and hyaluronic acid from fibroblasts and keratinocytes, respectively, were measured using ELISA. Expression levels of ceramide synthase 3 (CERS3), filaggrin (FLG), transglutaminase 1 (TGM1), and kallikrein-7 (KLK7) were evaluated by quantitative real-time PCR. Results: The wrinkle index was significantly reduced to $72.1 \%$ after a 6 -week of applying the PPE gel, along with a significant increase in skin hydration to $126.5 \%$. Type I collagen production from fibroblasts was enhanced slightly but significantly following treatment with PPE. PPE accelerated the expression of CERS3 (1.85-fold), FLG (1.35-fold), TGM1 (1.76-fold), and KLK7 (1.62-fold). Conclusion: Treatment with PPE alleviates wrinkle formation and simultaneously enhances skin hydration, which is induced via the accelerated expression of moisturizing-related proteins. These findings suggest that PPE is effective for combating dryness-induced wrinkle formation.
\end{abstract}

\section{Keywords}

Porcine Placenta, Wrinkle Formation, Moisturizing-Related Proteins, 


\section{Introduction}

As the skin is the outermost organ age-related changes such as wrinkles and pigmentation profoundly impact the quality of life, especially in women. Both extrinsic and intrinsic skin aging is known to occur with perpetual exposure to the external environment. Extrinsic skin aging primarily arises from ultraviolet (UV)-light exposure, directly enhancing reactive oxygen species, which induces the expression of matrix metalloproteinase (MMP)-1, MMP-3, and MMP-9. In contrast, intrinsically aged skin in UV-light-protected areas can be induced by the following factors: cell senescence with telomere shortening, mitochondrial DNA (mtDNA) mutations, oxidative stress, genetic mutations, and decreased levels of several hormones [1] [2] [3]. Wrinkle formation, which is a typical trait among age-related changes, can be observed in both UV-exposed and UV-protected sites. Based on previous studies, some underlying causes of wrinkle formation include reduced skin functions, skin dryness, UV exposure, and dermatitis [4] [5]. However, the treatment of wrinkles needs to be comprehensively addressed.

Clinical applications of human and/or porcine placenta extract (PPE) are widely accepted in oriental medicine as the placenta is a rich reservoir of diverse bioactive molecules. A previous study has shown that the injection of human placenta extract into wound boundaries accelerated decrease in wound size [6]. Moreover, oral administration of PPE reportedly reduces UVB-induced wrinkle formation in mice, along with the downregulation of MMP-2 mRNA expression [7]. In addition, studies using skin cells have revealed that human placenta extract accelerates keratinocyte and fibroblast proliferation [8] [9]. Accumulating evidence has highlighted the effects of placenta extract in the field of dermatology, and treatments with placenta are accepted as folk remedies to alleviate and prevent skin aging, especially in Asian countries where anti-aging is a major topic in dermato-cosmetology. However, it is necessary to elucidate the link between clinical improvement and molecular events induced by placenta extract. This study was designed to explore the effects of PPE on wrinkle formation, as well as to determine its underlying molecular mechanism.

\section{Materials and Methods}

\subsection{Preparation of Porcine Placenta Extract (PPE)}

After washing, the fresh porcine placenta underwent two freeze $\left(-20^{\circ} \mathrm{C}\right)$-thaw (under $10^{\circ} \mathrm{C}$ ) cycles. The obtained freeze-thaw drip was heated at $65^{\circ} \mathrm{C}$ for $6 \mathrm{~h}$ and subjected to vacuum-freeze drying, followed by removal of debris and filtration. Powdered PPE was reconstituted in sterilized water to a concentration of $50 \mathrm{mg} / \mathrm{mL}$ for in vitro testing. The gel formula containing $1.1 \mathrm{mg} / \mathrm{mL}$ PPE was kindly provided by Kasyu Industries Co., Ltd. (Fukuoka, Japan). 


\subsection{Evaluation of Elasticity, Water-Holding Property and Wrinkle Width}

The protocol was reviewed and approved by the Ethical Committee for Research in Septem-Soken. The study was designed as an open-labeled, single-blinded and randomized half-side investigation, without a control group. Fifteen female volunteers, recruited from our volunteer pool and aged 40 - 52 years (48.3 \pm 3.7$)$, participated in this study after informed consent was obtained from all volunteers. The volunteers were advised to apply a pearl-size amount (approximately $0.28 \mathrm{~g}$ ) of the PPE gel on the designated half side of the face, twice daily for six weeks. The wrinkle width, water-holding property, and elasticity were assessed using Visia Evolution (Canfield Scientific, Parsippany, NJ), Skicon-200EX (Yayoi, Tokyo, Japan), and Cutometer ${ }^{\circledR}$ MPA580 (Courage + Khazaka electronic GmbH, Cologne, Germany), respectively, at weeks 0 and 6 . The laboratory conditions were maintained in the temperature range of $20^{\circ} \mathrm{C}-22^{\circ} \mathrm{C}$, with a relative humidity of $50 \% \pm 5 \%$, during measurements. The data are expressed as the ratio (\%) to week 0 in the control and treated sites, respectively.

\subsection{Cells}

Normal human dermal fibroblasts (NHDFs) were obtained from PromoCell. NHDFs were maintained in fibroblasts growth media 2 (FGM2), supplemented with $20 \mu \mathrm{L} / \mathrm{mL}$ fetal calf serum, $1 \mathrm{ng} / \mathrm{mL}$ basic fibroblast growth factor (bFGF), and $5 \mu \mathrm{g} / \mathrm{mL}$ insulin at $37^{\circ} \mathrm{C}$ in a $5 \% \mathrm{CO}_{2}$-humidified atmosphere. The cells (F4-F6) were then subjected to experiments. $\mathrm{HaCaT}$ cells, an immortal human epidermal keratinocyte cell line, were maintained in $\mathrm{Ca}^{2+}$-free Dulbecco's Modified Eagle Medium (DMEM), supplemented with 5\% $\mathrm{Ca}^{2+}$-depleted FCS, $4 \mathrm{mM}$ glutamine, $1 \mathrm{mM}$ sodium pyruvate, and $2 \mathrm{mM} \mathrm{CaCl}_{2}$ at $37^{\circ} \mathrm{C}$ in a $5 \% \mathrm{CO}_{2}$-humidified atmosphere.

\subsection{Cytotoxicity}

In brief, $\mathrm{HaCaT}$ cells were inoculated into $96-$ well plates at a cell density of $5 \times$ $10^{3}$ cells/well and maintained in a $5 \% \mathrm{CO}_{2}$-humidified atmosphere at $37^{\circ} \mathrm{C}$ for 24 $\mathrm{h}$; then, the cells were further incubated in the presence of the indicated PPE concentrations. The neutral red assay was performed according to the manufacturer's instruction (Sigma-Aldrich, St. Louis, MO).

\subsection{Cell Proliferation}

In brief, $\mathrm{HaCaT}$ cells were seeded into 96-well plates at a cell density of $2 \times 10^{3}$ cells/well in a $5 \% \mathrm{CO}_{2}$-humidified atmosphere at $37^{\circ} \mathrm{C}$ for $24 \mathrm{~h}$. After incubation, the medium were removed. Then, $200 \mu \mathrm{L} /$ well of the medium containing the indicated concentration of PPE and predetermined concentration of BrdU was added to wells, and cells were subjected to further incubation for $48 \mathrm{~h}$. The incorporated BrdU was detected using a BrdU Cell Proliferation Assay kit (Cell Signaling, Boston, MA, USA), according to the manufacturer's instructions. 


\subsection{Extracellular Matrix Production}

To estimate the production of type I collagen from NHDFs and hyaluronic acid in HaCaT cells, NHDFs and HaCaT cells were seeded into 24-well plates at a cell density of $2.5 \times 10^{4}$ cells and maintained in a $5 \% \mathrm{CO}_{2}$-humidified atmosphere at $37^{\circ} \mathrm{C}$ for $24 \mathrm{~h}$. The cells were then treated with 5\% PPE for $24 \mathrm{~h}$. The amount of type I collagen and hyaluronic acid were measured in the supernatant using a type I collagen ELISA kit and hyaluronic acid ELISA kit (R\&D Systems, Minneapolis, MN), according to the manufacturer's instructions.

\subsection{RNA Isolation and Quantitative Real-Time PCR}

HaCaT cells were seeded into 6-well plates at a cell density of $3 \times 10^{5}$ cells/well and maintained in a $5 \% \mathrm{CO}_{2}$-humidified atmosphere at $37^{\circ} \mathrm{C}$ for $24 \mathrm{~h}$; then, the cells were further cultivated in the presence of KX-1 or its fractions for $24 \mathrm{~h}$. Cells were harvested and total RNA was prepared using an SV RNA isolation kit (Promega, Madison, WI, USA), according to the manufacturer's instructions, followed by reverse transcription with ReverTra Ace ${ }^{\varpi}$ qCR RT Master Mix (TOYOBO, Osaka, Japan). PCR amplification and detection were performed on a CFX96 real-time PCR system (BioRad, Hercules, CA, USA) using a KAPA SYBR FAST qPCR master mix (KAPA Biosystems, Woburn, MA, USA). The following primer pairs were used: $\beta$-actin, 5'-GATGAGATTGGCATGGCTTT-3' (sense) and 5'-CACCTTCACCGTTCCAGTTT-3' (antisense); CERS3, 5'-ACAT TCCACAAGGCAACCATTG-3' (sense) and 5'-CTCTTGATTCCGCCGACTCC-3' (antisense); FLG, 5'-TGGCAGCTATGGTAGTGCAG-3' (sense) and 5'-CCAAA CGCACTTGCTTTACA-3' (antisense); TGM1, 5'-ACTACGGCCAGTTTGACC AC-3' (sense) and 5'-TCGGGAGTAATCACCAGACC-3' (antisense); and KLK7, 5'-ATGGCAAGATCCCTTCTCCT-3' (sense) and 5'-GGCGCCATCAATAATC TTGT-3' (antisense). Target mRNA expression was quantified using the comparative threshold cycle $(\mathrm{Ct})$ method for relative quantification $\left(2^{-\delta \delta \mathrm{Ct}}\right)$, normalized to the geometric mean of the reference gene $\beta$-actin.

\subsection{Statistical Analysis}

Data are expressed as the mean \pm standard deviation (SD). Statistical analysis was performed using Student $\mathrm{t}$-test. A value of $p<0.1$ was considered statistically significant for in vivo tests. A value of $p<0.05$, was assumed to be significant for in vitro tests.

\section{Results}

\subsection{Changes in Skin Properties}

To evaluate the effects of PPE on skin conditions, various skin properties were assessed. The number of detectable wrinkles was reduced (Figure 1(a)) and the calculated wrinkle index was also significantly decreased to $72.1 \%$ of the control (Figure 1(b)). In contrast, conductance was significantly increased to $126.5 \%$ at 
the PPE-treated site, when compared with the control site (Figure 1(c)); no significant changes in gross elasticity (Figure $1(\mathrm{~d})$ ) and elastic recovery (Figure $1(\mathrm{e}))$ were observed.

\subsection{Cytotoxicity and Cell Proliferation}

The neutral red assay was used to estimate the cytotoxicity of PPE. The results revealed that PPE did not significantly affect cell viability at concentrations ranging between 1 and 5\% of PPE (Figure 2(a)). Likewise, no significant effects on cell proliferation were observed at $2 \%$ and $5 \%$ of PPE (Figure 2(b)). Thus, a concentration of 5\% PPE was used for further experiments.

\subsection{Extracellular Matrix Productions}

To estimate the effects of PPE on extracellular matrix production, the production of type I collagen and hyaluronic acid was assessed in NHDF. Treatment with PPE induced a marginal but significant enhancement in type I collagen production (Figure 3(a)). In contrast, PPE did not impact hyaluronic acid production (Figure 3(b)).

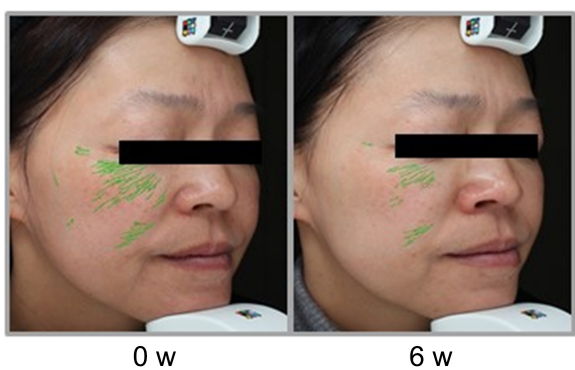

(a)

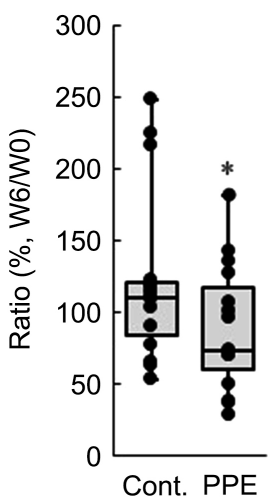

(b)

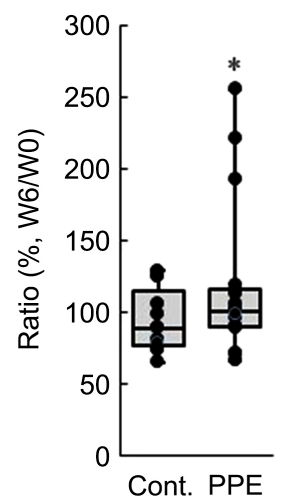

(c)

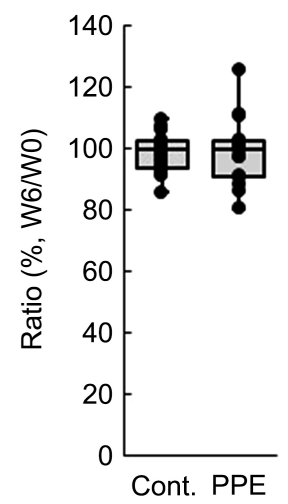

(d)

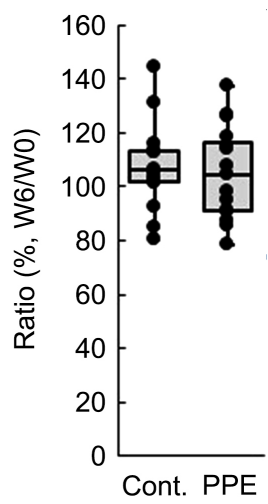

(e)

Figure 1. Changes in skin appearance and properties by PPE treatment. (a) A representative image of wrinkles (green lines) on the right cheek; (b) Comparison of calculated wrinkle index between the PPE treated site and the control site. A significant reduction in the wrinkle index can be observed on the PPE-treated site. ${ }^{*} p<0.05$; (c) Comparison of skin hydration between the PPE-treated site and control site. A significant increase in skin hydration can be observed on the PPE-treated site. ${ }^{\star} p<0.05$; (d) and (e) Comparison of gross elasticity and elastic recovery between the PPE treated site and the control site. No significant changes can be observed. PPE, porcine placenta extract. 


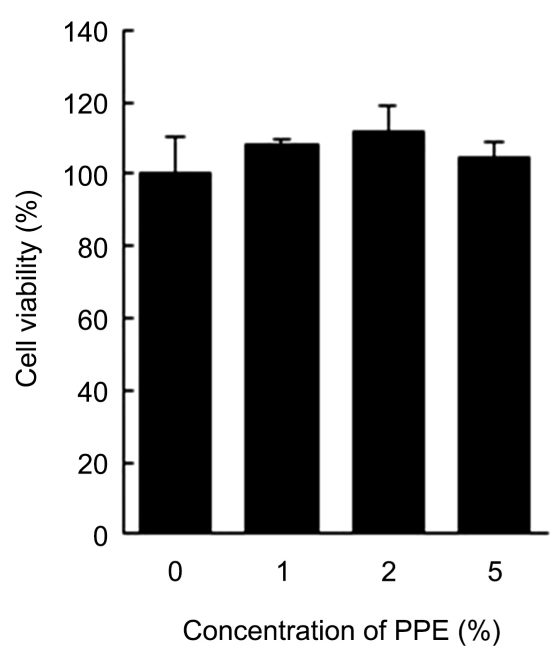

(a)

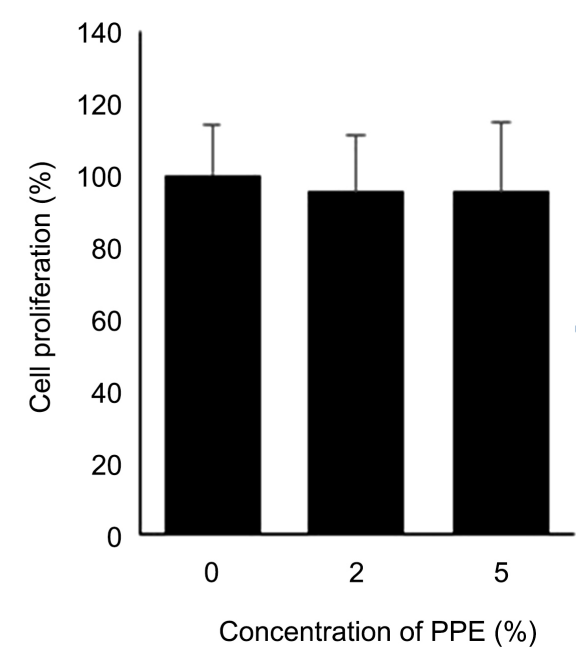

(b)

Figure 2. The cytotoxicity of PPE and the effect on cell proliferation. (a) Cytotoxicity of PPE. No significant cytotoxicity can be detected in the concentration range employed in this experiment; (b) The effect of PPE on keratinocyte proliferation. PPE treatment does not affect cell proliferation in the concentration range employed in this experiment. PPE, porcine placenta extract.

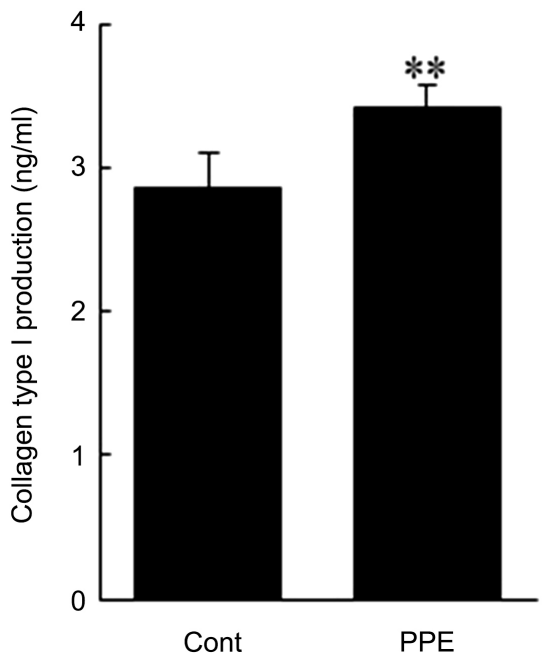

(a)

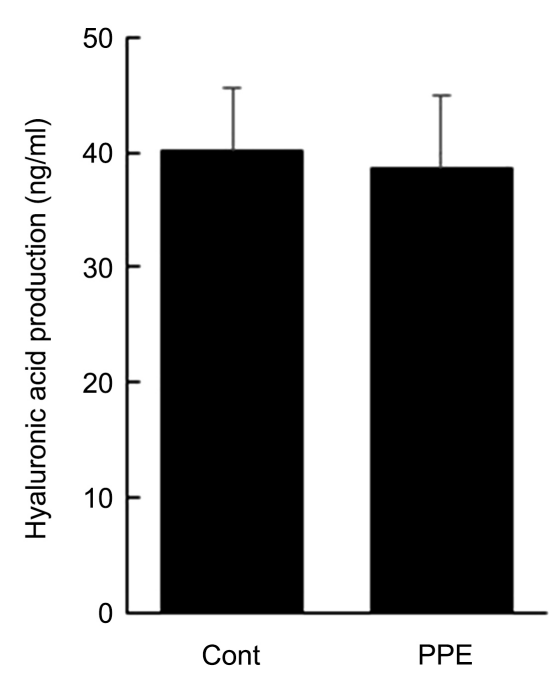

(b)

Figure 3. The effects of PPE on the extracellular matrix. (a) Type I collagen production from NHDFs. PPE significantly enhances type I collagen production. ${ }^{\star *} p<0.01$; (b) Hyaluronic acid production in $\mathrm{HaCaT}$ cells. No significant change can be observed. PPE, porcine placenta extract.

\subsection{Expression of Proteins in HaCaT Cells}

Next, we evaluated the effects of PPE on the mRNA expression of proteins involved in skin barrier function and water-holding properies. The mRNA expressions levels of ceramide synthase 3 (CERS3), filaggrin (FLG), transglutaminase 1 (TGM1) and kallikrein-7 (KLK7) were significantly upregulated to $1.85,1.35$, 1.76, and 1.62, respectively (Figures $4(\mathrm{a})-(\mathrm{d})$ ). 


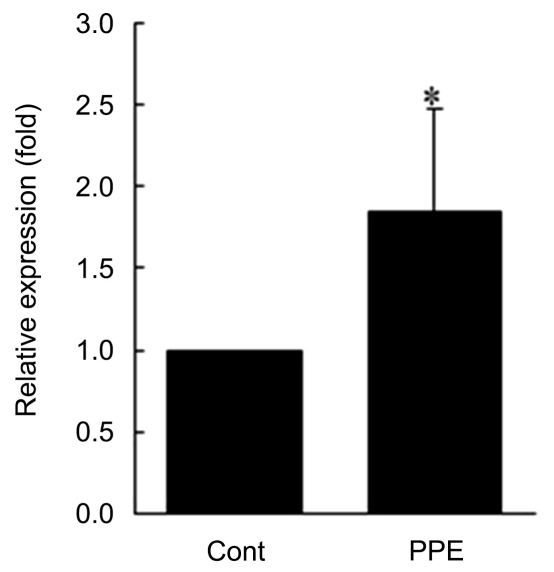

(a)

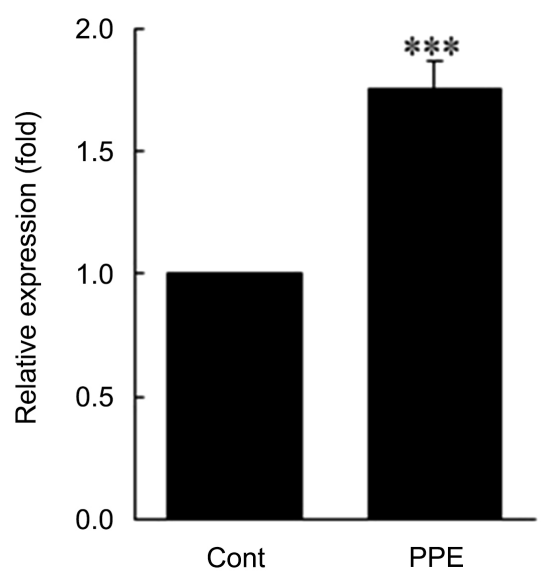

(c)

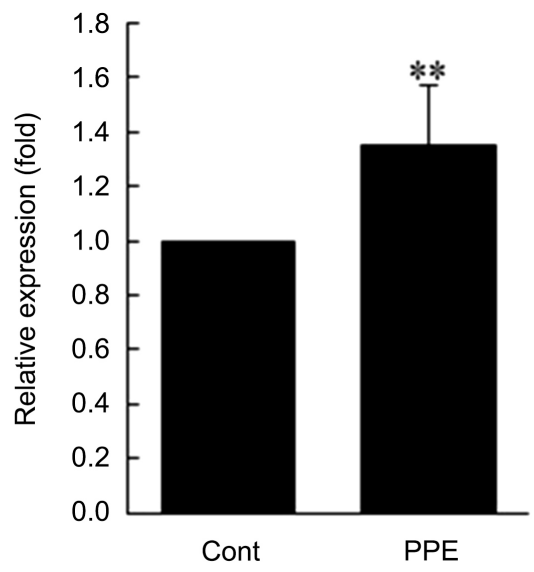

(b)

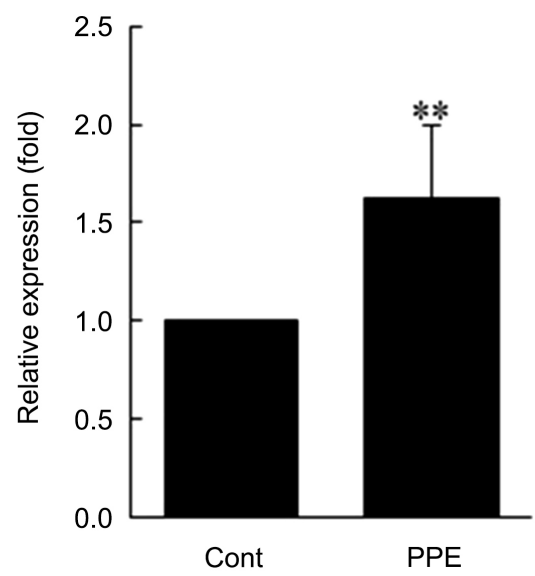

(d)

Figure 4. The effects of PPE on mRNA expressions. (a) CERS3 mRNA expression. PPE accelerated mRNA expression of CERS3. ${ }^{*} p<0.05$; (b) FLG mRNA expression. PPE accelerates mRNA expression of FLG. ${ }^{* *} p<0.01$; (c) TGM1 mRNA expression. PPE accelerated mRNA expression of TGM1. ${ }^{* *} p<0.001$; (d) KLK7 mRNA expression. PPE accelerated mRNA expression of KLK7. ${ }^{* *} p<0.01$. PPE, porcine placenta extract; CERS3, ceramide synthase 3; FLG, filaggrin; TGM1, transglutaminase; KLK7, kallikrein-7.

\section{Discussion}

In the skin, extrinsic and intrinsic skin aging occurs owing to perpetual exposure to the external environment. Wrinkles, which are typical among age-related changes, are caused by extrinsic and intrinsic factors, as reported previously [1] [2] [3] [4] [5]. However, the treatment of wrinkles needs to be further investigated.

Herein we revealed that PPE alleviated facial wrinkle formation (Figure 1(a) and Figure 1(b)). As previous studies have reported that wrinkle formation coincides with reduced hydration and elasticity levels [10] [11], we focused on epidermal water-holding properties and skin elasticity. In the present study, PPE increased the water content of the epidermis (Figure 1(c)). In contrast, skin elasticity indices were not affected following treatment with PPE (Figure 1(d) and Figure 1(e)), although PPE enhanced type I collagen production from fibrob- 
lasts (Figure 3(a)). These results suggest that the molecular mechanism underlying the reduction of wrinkle parameters is dependent on events in the epidermis, but not in the dermis. As various molecules such as hyaluronic acid, ceramides and natural moisturizing factors contribute to the water content in the epidermis, we evaluated the effect of PPE on the production of hyaluronic acid which is secreted from keratinocytes [12]. Hyaluronic acid secretion from HaCaT cells was not impacted by PPE (Figure 3(b)). Next, we examined the mRNA expression of CERS3, FLG, TGM1 and KLK7. As shown in Figure 4, PPE treatment accelerated all mRNA expression levels. CERS3 is predominantly expressed in keratinocytes and synthesizes C24-ceramides and ceramides with longer acyl chains [13]. Additionally, FLG is expressed in the granular layer of the epidermis and is ultimately degraded into free amino acids, which provide a natural moisturizing factor [14]. Therefore, PPE treatment may directly potentiate the skin barrier function and water-holding properties by accelerating the expressions of CERS3 and FLG mRNA. Conversely, it is postulated that well-balanced regulation of keratinocyte differentiation and corneocyte exfoliation is crucial for the construction of a healthy stratum corneum. TGM1 contributes to the assembly of the cornified envelope [15] and KLK7 plays a role in shedding corneocytes [16], indicating that these enzymes are pivotal participants in the formation of a healthy stratum corneum. As PPE accelerated the expression of TGM1 and KLK7, treatment with PPE is speculated to involve the construction of a healthy stratum corneum. Collectively, these results suggest that PPE is involved in restoring the skin barrier function and water-holding properties. In conclusion, treatment with PPE is effective for dryness-induced wrinkle formation via enhancement of skin hydration.

\section{Acknowledgements}

We would like to thank Editage (https://www.editage.com/) for English language editing.

\section{Conflicts of Interest}

The author declares no conflicts of interest regarding the publication of this paper.

\section{References}

[1] Kohl, E., Steinbauer, J., Landthaler, M. and Szeimies, R.M. (2011) Skin Ageing. Journal of the European Academy of Dermatology and Venereology, 25, 873-884. https://doi.org/10.1111/j.1468-3083.2010.03963.x

[2] Naylor, E.C., Watson, R.E.B. and Sherratt, M.J. (2011) Molecular Aspects of Skin Ageing. Maturitas, 69, 249-256. https://doi.org/10.1016/j.maturitas.2011.04.011

[3] Makrantonaki, E. and Zouboulis, C.C. (2007) Characteristics and Pathomechanisms of Endogenously Aged Skin. Dermatology, 214, 352-360. https://doi.org/10.1159/000100890

[4] Langton, A.K., Sherratt, M.J., Griffiths, C.E.M. and Watson, R.E. (2010) A New 
Wrinkle on Old Skin: The Role of Elastic Fibres in Skin Ageing. International Journal of Cosmetic Science, 32, 330-339. https://doi.org/10.1111/j.1468-2494.2010.00574.x

[5] Hashizume, H. (2004) Skin Aging and Dry Skin. The Journal of Dermatology, 31, 603-609. https://doi.org/10.1111/j.1346-8138.2004.tb00565.x

[6] Hong, J.W., Lee, W.J., Hahn, S.B., Kim, B.J. and Lew, D.H. (2010) The Effect of Human Placenta Extract in a Wound Healing Model. Annals of Plastic Surgery, 65, 96-100. https://doi.org/10.1097/SAP.0b013e3181b0bb67

[7] Hong, K.B., Park, Y., Kim, J.H., Kim, J.M. and Suh, H.J. (2015) Effects of Porcine Placenta Extract Ingestion on Ultraviolet B-induced Skin Damage in Hairless Mice. Korean Journal for Food Science of Animal Resources, 35, 413-420. https://doi.org/10.5851/kosfa.2015.35.3.413

[8] O’Keefe, E.J., Payne, R.E. and Russell, N. (1985) Keratinocyte Growth-Promoting Activity from Human Placenta. Journal of Cellular Physiology, 124, 439-445 https://doi.org/10.1002/jcp.1041240312

[9] Cho, H.R., Ryou, J.H., Lee, J.W. and Lee, M.H. (2008) The Effects of Placental Extract on Fibroblast Proliferation. Journal of Cosmetic Science, 59, 195-202

[10] Marini, A., Grether-Beck, S., Jaenicke, T., Weber, M., Burki, C., Formann, P., Brenden, H., Schönlau, F. and Krutmann, J. (2012) Pycnogenol ${ }^{\circledR}$ Effects on Skin Elasticity and Hydration Coincide with Increased Gene Expressions of Collagen Type I and Hyaluronic acid Synthase in Women. Skin Pharmacology and Physiology, 25, 86-92. https://doi.org/10.1159/000335261

[11] Choi, J.W., Kwon, S.H., Huh, C.H., Park, K.C. and Youn, S.W. (2013) The Influences of Skin Visco-Elasticity, Hydration Level and Aging on the Formation of Wrinkles: A Comprehensive and Objective Approach. Skin Research and Technology: Official Journal of International Society for Bioengineering and the Skin, 19, e349-e355. https://doi.org/10.1111/j.1600-0846.2012.00650.x

[12] Sakai, S., Yasuda, R., Sayo, T., Ishikawa, O. and Inoue, S. (2000) Hyaluronan Exists in the Normal Stratum Corneum. Journal of Investigative Dermatology, 114, 1184-1187. https://doi.org/10.1046/j.1523-1747.2000.00992.x

[13] Levy, M. and Futerman, A.H. (2010) Mammalian Ceramide Synthases. IUBMB Life, 62, 347-356. https://doi.org/10.1002/iub.319

[14] Brettmann, E.A. and de Guzman Strong, C. (2018) Recent Evolution of the Human Skin Barrier. Experimental Dermatology, 27, 859-866.

https://doi.org/10.1111/exd.13689

[15] Eckert, R.L., Sturniolo, M.T., Broome, A.M., Ruse, M. and Rorke, E.A. (2005) Transglutaminase Function in Epidermis. Journal of Investigative Dermatology, 124, 481-492. https://doi.org/10.1111/j.0022-202X.2005.23627.x

[16] Caubet, C., Jonca, N., Brattsand, M., Guerrin, M., Bernard, D., Schmidt, R., Egelrud, T., Simon, M. and Serre, G. (2004) Degradation of Corneodesmosome Proteins by Two Serine Proteases of the Kallikrein Family, SCTE/KLK5/hK5 and SCCE/KLK7/hK7. Journal of Investigative Dermatology, 122, 1235-1244.

https://doi.org/10.1111/j.0022-202X.2004.22512.x 